\title{
Évolution du délai d'établissement du voisement (VOT) dans le vieillissement sain entre 40 et 80 ans
}

\author{
Clémence Verhaegen* 1 , Véronique Delvaux**, Kathy Huet**, Myriam \\ Piccaluga***, Charlotte Vanderwaele, Bernard Harmegnies** \\ * Métrologie et Sciences du Langage, Université de Mons - Belgique \\ **Université de Mons - Belgique \\ ***Institut de Recherche en Sciences et Technologies du Langage, Mons, Belgium
}

Résumé. Cette étude s'intéresse à l'évolution des capacités de production de la parole dans le vieillissement, dans une perspective lifespan. En effet, en plus de la diminution des capacités de production langagière, l'âge entraîne une réduction des capacités de contrôle moteur de la parole et une dégradation des structures oro-laryngées. Cependant, les effets de l'âge sur les capacités de production de la parole restent peu étudiés, particulièrement en langue française. Dans cette étude, nous nous centrons sur l'analyse du délai d'établissement du voisement (VOT), un paramètre important de la distinction entre les occlusives voisées et non voisées en français, et qui constitue un indice intéressant des capacités de coordination entre les gestes glottiques et supra-glottiques. Dans ce but, nous avons proposé une tâche de répétition de non-mots CVCV, comprenant les six consonnes occlusives du français $/ \mathrm{p}, \mathrm{t}, \mathrm{k}, \mathrm{b}, \mathrm{d}, \mathrm{g} /$ à des participants de 40 à 80 ans, répartis en quatre groupes par tranches d'âges de 10 ans. Les résultats montrent la présence d'une diminution des valeurs de VOT ainsi que des pourcentages de voisement des premières consonnes voisées des non-mots après 60 ans. La présence d'effets délétères de l'âge sur les capacités d'initiation et de maintien du voisement des voisées du français est discutée.

\begin{abstract}
The effects of aging on the production of VOT between 40 and 80 years old. This study explores the effects of aging on speech production capacities, in a lifespan perspective. Indeed, besides the agerelated effects on language production capacities, some studies found deleterious effects of aging on speech motor control and oro-laryngeal structures. However, the age-related effects on speech production capacities remain rarely explored, especially in French. In this study, we focus on the analyses of voice onset time (VOT), an interesting cue of coordination capacities between glottal and supra-glottal articulators. For that purpose, we propose a non-words repetition task including the six French voiceless and voiced stop consonants $/ \mathrm{p} /, / \mathrm{t} /, / \mathrm{k} /, / \mathrm{b} /, / \mathrm{d} /, / \mathrm{g} /$ to 4 groups of participants of 10 years each, between 40 and 80 years old. The results show an age-related decrease on the VOT values and the percentages of voicing of the first voiced stops of the non-words after 60 years of age. The deleterious effects of aging on the capacities to initiate and maintain voicing of French voiced stops is discussed.
\end{abstract}




\section{Introduction}

Comme beaucoup d'aspects de la cognition, les capacités de production du langage sont affectées par le vieillissement (Baltes, Staudinger, \& Lindenberger, 1999; Burke \& Shafto, 2004). Le nombre de personnes âgées ayant tendance à croître à l'heure actuelle au niveau de la population mondiale, étudier les effets du vieillissement sur ces capacités constitue un challenge important, tant au niveau de la recherche qu'au niveau de la clinique.

De nombreuses études ont démontré des effets de l'âge sur les capacités de production du langage (Burke, MacKay, Worthley, \& Wade, 1991; Burke \& Shafto, 2004; Connor, Spiro, Obler, \& Albert, 2004; Verhaegen \& Poncelet, 2013). Par contre, les effets de l'âge sur les capacités de production de la parole restent peu étudiés, particulièrement dans une population francophone. Or, certaines études ont montré que l'âge entraînait une dégradation des capacités de contrôle moteur de la parole, ainsi que de nombreux changements au niveau des structures articulatoires impliquées dans la production de la parole, telles qu'une ossification des cartilages laryngés, une atrophie des muscles du larynx, des lèvres et de la langue, ainsi qu'une dégradation tissulaire. Ces changements entraîneraient une diminution de la fluence verbale et une augmentation des erreurs de répétition de mots et de non-mots (Aoki \& Fukuoa, 2010; Bilodeau-Mercure, Lortie, Sato, Guitton, \& Tremblay, 2015; Krampe, Mayr, \& Kliegl, 2005; Linville, 2000; Schötz, 2007; Torre \& Barlow, 2009; Tremblay, Sato, \& Deschamps, 2017).

Dans la littérature portant sur les patients cérébrolésés, de nombreux auteurs se sont intéressés à un indice acoustique, fréquemment altéré en cas de difficultés de contrôle de la parole : le délai d'établissement du voisement (généralement dénommé «VOT », de l'anglais Voice Onset Time). Au sens de Lisker \& Abramson (1964), le VOT est le délai (en millisecondes) entre le relâchement de l'occlusion supra-glottique et l'apparition des vibrations laryngées. Sa valeur est positive lorsque l'apparition du signal glottique suit la levée de l'occlusion et négatif dans le cas contraire. Il est un important paramètre de l'opposition entre les occlusives sourdes et sonores dans un grand nombre de langues, dont le français (Cho \& Ladefoged, 1999; Serniclaes, 1987). Mais dans des langues différentes, les frontières catégorielles s'établissent de manière diverse : si le VOT des réalisations rend bien compte de la prise en compte par le locuteur du trait de voisement, il ne le fait pas de la même manière dans toutes les langues. Ainsi, par exemple, en anglais, le VOT est positif et court dans la plupart des cas pour les voisées et positif mais long dans la plupart des cas pour les non-voisées. En français, par contre, il est négatif pour les voisées et positif pour les non-voisées. Le VOT constitue en outre un indice du contrôle des relations temporelles et de la coordination entre les gestes glottiques et supra-glottiques, ce qui en fait une mesure d'élection pour l'étude des troubles de la planification et de l'exécution motrice des sons de parole. Les études réalisées auprès de patients présentant une aphasie, une dysarthrie ou une apraxie de la parole, montrent que les patients présentent des durées de VOT plus courtes ou plus variables pour les occlusives (Auzou et al., 2000; Baqué, Marczyk, Rosas, \& Estrada, 2015; Marczyk, Baqué, Rosas, \& Nespoulous, 2011; Nespoulous, Baqué, Rosas, Marczyk, \& Estrada, 2013; Ryalls, Provost, \& Arsenault, 1995; Verhaegen et al., 2019).

Dans ce contexte étant donné que le vieillissement sain aurait un effet délétère sur le contrôle de la parole, il est intéressant de se demander dans quelle mesure, en français, le VOT serait affecté par ce paramètre. À l'heure actuelle, les études portant sur ce sujet restent peu nombreuses - particulièrement en langue française- et peu contributives, étant donné la grande variabilité interindividuelle. Certaines recherches, réalisées en langue anglaise, ont montré la présence de valeurs de VOT plus courtes pour les consonnes occlusives chez les personnes de plus de 60 ans, en comparaison avec des participants de moins de 30 ans (Gates et al., 2010; Morris, McCrea, \& Herring, 2008; Xuanda, Ziyu, \& Jian, 2018), alors que d'autres études n'ont pas montré de différence significative, mais ont 
cependant indiqué une plus grande variabilité au niveau des valeurs des VOT, menant parfois à un chevauchement entre les valeurs pour les consonnes occlusives voisées et non voisées (Schötz, 2007; Sweeting \& Baken, 1982; Torre \& Barlow, 2009). Les auteurs attribuent ces résultats à des difficultés de coordination entre les articulateurs chez les participants plus âgés. Les études réalisées jusqu'aujourd'hui portent principalement sur les participants anglophones. Or, comme on l'a souligné précédemment, le français a la particularité de présenter des valeurs de VOT négatives pour les occlusives voisées, le voisement apparaissant avant l'explosion. Ce prévoisement est relativement long, de -100 à -70 ms environ (Serniclaes, 1987). La tenue de celui-ci nécessite une coordination précise entre les articulateurs glottiques et supra-glottiques, qui peut dès lors être altérée chez des participants présentant potentiellement des difficultés de contrôle et de coordination de la parole, telles que les personnes âgées. D'une part, en initiale absolue, les événements que sont le déclenchement du voisement et la levée de l'occlusion doivent être générés en ordre chronologique inverse pour les occlusives voisées (déclenchement du voisement suivi de la levée de l'occlusion) et non voisées (levée de l'occlusion suivie du déclenchement du voisement) ; en anglais, par contre, l'ordre est invariant (levée de l'occlusion suivie du déclenchement du voisement). D'autre part, le déclenchement et la tenue du prévoisement des voisées doivent s'effectuer pendant la phase occlusive, ce qui met, pendant la durée de la tenue, les cavités supraglottiques en surpression, au prix d'un effort non négligeable ; en anglais, par contre, c'est toujours après la levée de l'occlusion que le voisement est déclenché, ce qui ne nécessite aucun effort plus particulier pour les voisées. En langue française, une étude réalisée par Ryalls, Cliche, Fortier-Blanc, Coulombe, et Prud'hommeaux (1997) a montré que les personnes de plus de 60 ans présentaient des valeurs de VOT plus variables pour les occlusives voisées que les participants de 20 à 30 ans. Les auteurs font l'hypothèse que cela serait dû au fait que certains des participants de l'étude présenteraient des valeurs de VOT plus courtes pour les voisées, en raison de difficultés à initier le voisement de ces consonnes. Cependant, les auteurs indiquent que les résultats nécessiteraient d'être confirmés dans d'autres études, en raison notamment du faible nombre de participants $(N=10)$.

Enfin, beaucoup d'études sur les effets du vieillissement tendent à mettre en contraste des comportements de sujets réputés "jeunes » avec des comportements de sujets considérés comme « âgés ». Souvent, on voit ainsi opposer des groupes dont les sujets ont moins de 30 ans avec des groupes de sujets ayant atteint la soixantaine. Cette stratégie peut se justifier si, dans une étude exploratoire, on souhaite tester la dépendance d'une variable donnée à l'âge. Mais si cette dépendance est établie ou très vraisemblable, il peut être au contraire intéressant d'en investiguer les ressorts dans une dynamique plus continue que dichotomique, en examinant les effets de l'âge chez des participants sur plusieurs décennies différentes, afin d'observer dans quelle mesure l'éventuel déclin s'effectue ou non de façon progressive, même avant 60 ans, comme cela est le cas pour les troubles de la production des mots liée à l'âge (Burke \& Shafto, 2004; Connor et al., 2004; Verhaegen \& Poncelet, 2013).

Dans cette étude, nous nous intéressons à la variation, sous l'effet de l'âge, du VOT des consonnes occlusives chez les locuteurs francophones. Dans ce but, nous avons présenté une tâche de répétition de non-mots comprenant les six consonnes occlusives orales voisées et non voisées du français /p,t,k,b,d,g/ associées aux trois voyelles cardinales /a,i,u/. Nous avons présenté cette tâche à des participants de 40 à 80 ans, par tranches d'âges de 10 ans, afin d'observer les effets de l'âge sur les capacités de production de la parole dans une perspective « lifespan ». Dans une langue telle que le français, dans laquelle les occlusives voisées comportent un prévoisement long, nous nous attendions à ce que l'âge entraîne des difficultés de contrôle et de coordination entre les articulateurs glottiques et supraglottiques, qui se marqueraient par des difficultés d'initiation et de maintien du prévoisement des voisées. Dans nos analyses acoustiques, nous supposions dès lors une 
diminution progressive avec l'âge, à la fois au niveau de la durée des VOT des occlusives voisées ainsi qu'au niveau du pourcentage de voisement de ces consonnes. Nous nous attendions à ce que cela touche particulièrement la première consonne lorsqu'elle est voisée, étant donné que le voisement serait déjà initié en seconde consonne par la voyelle précédente.

\section{Méthode}

\subsection{Participants}

Quarante-sept sujets ont participé à notre étude. Ils se répartissent en quatre groupes d'âge : (1) 10 participants âgés de 40 à 49 ans, (2) 13 participants âgés de 50 à 59 ans, (3) 12 participants entre 60 et 69 ans et (4) 12 participants entre 70 et 79 ans. Tous les participants étaient de langue maternelle française exempts de troubles du langage, neuropsychologiques ou psychiatriques, ainsi que de troubles de la vision non corrigés. Les participants utilisant une médication risquant d'affecter leurs fonctions cognitives (tels que les antidépresseurs) ont été exclus de l'étude. Les participants présentant des signe de démence (évaluée à l'aide de l'échelle de la démence de Mattis, Schmidt, Freidl, Fasekas, Reinhart, \& Grieshofer, 1994) ont été écartés. Les données démographiques sont présentées dans le Tableau 1.

En vue d'éventuellement exclure également les participants qui présenteraient des altérations du langage, des fonctions exécutives ou de l'audition plus importants que dans la population âgée saine, nous avons soumis aux participants des tâches langagières, exécutives et de mémoire à court terme et leur avons administré un audiogramme (voir Tableau 1). La tâche de dénomination d'images issue de la batterie Lexis (Bilocq, De Partz, De Wilde, Pillon, \& Seron, 2001) se composait de 80 images. Une analyse de variance (ANOVA) effectuée sur le taux de réponses correctes dans les 4 groupes de participants indique un effet de l'âge, $F(3,2257)=317.68, p<.001$. Le test post-hoc de Tuckey $(p<.05)$ montre que les personnes de plus de 70 ans présentent des résultats plus faibles que les participants plus jeunes. En ce qui concerne les capacités de compréhension du langage, les résultats des sujets dans une tâche d'appariement phrases/images (MT-86, Joanette, Nespoulous, \& Roch Lecours, 1998) montrent un effet significatif de l'âge, $F(3,2257)=$ $52.33, p<.001$. Le test post-hoc de Tuckey $(p<.05)$ révèle que les sujets de plus de 70 ans présentent des résultats plus faibles que les personnes plus jeunes. Les capacités de mémoire verbale à court terme et exécutives ont été évaluées à l'aide de tâches minimisant les influences des capacités langagières sur les résultats (Quinette et al., 2014). Les tâches de mémoire verbale à court terme et de mémoire de travail consistaient respectivement en des tâches d'empan de chiffres endroit et envers. Dans ces tâches, les participants entendaient des chiffres, dont le nombre croissait entre les niveaux, et il leur était demandé de les répéter dans l'ordre ou dans l'ordre inverse respectivement, à l'aide de cartons représentant les chiffres entendus. La tâche d'inhibition consistait en une planche comportant des figures sans signification contenant des courbes ou des lignes droites, de couleur noire ou rouge. Dans cette tâche, les participants devaient répondre « oui » en cas de figure comportant des lignes courbes, «non » lorsque les figures comportaient des lignes droites et toujours «non » lorsque la figure était rouge. Dans la tâche de flexibilité, les participants devaient relier alternativement des cercles et des carrés à l'aide d'un crayon. Enfin, dans la tâche de mise à jour, les sujets recevaient des planches comportant des figures sans signification, dont le nombre augmentait à chaque essai. Il leur était demandé de pointer une figure différente à chaque nouvelle planche. Les résultats indiquent des taux de réponses correctes plus faibles avec l'âge dans la tâche de mémoire à court terme, $F(3,2257)=436.85, p<.001$, de mémoire de travail, $F(3,2257)=301.73, p<.001$, 
d'inhibition, $F(3,2257)=67.46, p<.001$, de mise à jour, $F(3,2257)=74.50, p<.001$, et de flexibilité, $F(3,2257)=220.47, p<.001$. Dans les tâches de mémoire à court terme et de travail ainsi que de flexibilité, les tests post-hoc de Tuckey $(p<.05)$ montrent que les personnes âgées de plus de 70 ans présentent des performances plus faibles que les personnes plus jeunes. Dans la tâche de mise à jour, les résultats diminuent dès 60 ans. Dans la tâche d'inhibition, les résultats sont légèrement plus faibles chez les participants de 40 à 49 ans et 70-79 ans que dans les autres groupes. Au niveau de l'audition, les participants ont été évalués à l'aide d'une audiométrie tonale liminaire au casque. L'ANOVA effectuée sur la moyenne des deux oreilles sur les fréquences de 500, 1000, 2000 et $4000 \mathrm{~Hz}$ montrent un effet de l'âge, $F(3,2257)=895.27, p<.001$, après 70 ans (Tuckey, $p<.05$ ).

En résumé, ces résultats indiquent des performances significativement plus faibles après 70 ans. Celles-ci ne sont pas plus importantes que celles observées dans le vieillissement sain dans d'autres études. En effet, en ce qui concerne le langage, la mémoire à court terme et les fonction exécutives, les recherches indiquent un déclin d'au moins 15 à 25 pourcents des performances après 70 ans (Baltes et al., 1999; Belleville, Rouleau, \& Caza, 1998; Burke \& Shafto, 2004; Connor et al., 2004; Maylor, Vousden, \& Brown, 1999; Oberauer, 2001; Salthouse, 2009; Verhaegen \& Poncelet, 2013). Au niveau du déclin des capacités auditives avec l'âge, les études indiquent une dégradation des capacités de 3 à $5 \mathrm{~dB}$ par décennie entre 30 et 60 ans et entre 10 à $15 \mathrm{~dB}$ après 60 ans. Après 70 ans, les troubles auditifs liés à l'âge affecteraient 100 pourcents des personnes âgées (Cruickshanks et al., 1998; Surprenant, 2007).

Cet examen très fouillé des caractéristiques de nos sujets nous permet, en bref, de conclure que l'échantillon que nous avons retenu est bien conforme à ce que l'on attend de personnes représentant l'avancée naturelle en âge dans une dynamique de vieillissement sain au plein du terme.

Tableau 1. Caractéristiques de l'échantillon

\begin{tabular}{|c|c|c|c|c|}
\hline & $40-49$ ans & 50-59 ans & 60-69 ans & $70-79$ ans \\
\hline $\begin{array}{l}\text { Nombre de participants (proportion } \\
H / F \text { ) }\end{array}$ & $10(4 / 6)$ & $13(4 / 9)$ & $12(3 / 9)$ & $12(4 / 8)$ \\
\hline Âge & $44.41(3.25)$ & $53.85(2.80)$ & $63.25(2.58)$ & $74.50(3.28)$ \\
\hline \multicolumn{5}{|l|}{ Évaluation langagière } \\
\hline Dénomination $(\%)$ & $93.31(6.75)$ & $88.69(3.89)$ & $92.32(4.35)$ & $83.33(9.08)$ \\
\hline Appariement phrases/images (\%) & $100.00(0)$ & $100.00(0)$ & $99.56(1.52)$ & $99.34(1.64)$ \\
\hline \multicolumn{5}{|l|}{ Evaluation Cognitive } \\
\hline Mémoire à court terme $(\%)$ & $50.69(10.33)$ & $53.21(9.94)$ & $54.17(10.95)$ & $34.03(13.04)$ \\
\hline Mémoire de travail $(\%)$ & $45.14(12.54)$ & $40.38(10.11)$ & $47.92(12.87)$ & $30.56(8.94)$ \\
\hline Flexibilité (nb. erreurs) & $0.00(0)$ & $0.00(0)$ & $0.08(0.29)$ & $1.00(1.59)$ \\
\hline Mise à jour (\%) & $93.51(7.43)$ & $86.32(8.67)$ & $89.81(9.12)$ & $88.88(6.70)$ \\
\hline Inhibition (\%) & $97.76(5.14)$ & $98.73(2.42)$ & $99.52(1.11)$ & $97.38(2.99)$ \\
\hline $\begin{array}{l}\text { Evaluation auditive } \\
\text { (audiométrie tonale, moyenne des } 2 \\
\text { oreilles, dB HL) }\end{array}$ & $21.56(7.58)$ & $11.99(5.00)$ & $19.14(5.02)$ & $28.80(5.67)$ \\
\hline
\end{tabular}

\subsection{Tâche expérimentale}

Pour cette tâche, créée dans notre laboratoire, notre intérêt s'articulant autour du trait de voisement en langue française, nous avons choisi 48 non-mots CVCV, comprenant toutes les occlusives voisées et non voisées du français $/ \mathrm{p}, \mathrm{t}, \mathrm{k}, \mathrm{b}, \mathrm{d}, \mathrm{g} /$, ainsi que les 3 voyelles 
cardinales /a,i,u/. Pour l'environnement vocalique d'aperture maximale (/a/), toute le combinatoire consonantique possible a été exploitée (36 non-mots $\mathrm{C}_{1} \mathrm{~V}[\mathrm{a}] \mathrm{C}_{2} \mathrm{~V}$ [a] où $\mathrm{C}_{1}=$ /p,t,k,b,d,g/ et $\mathrm{C}_{2}=/ \mathrm{p}, \mathrm{t}, \mathrm{k}, \mathrm{b}, \mathrm{d}, \mathrm{g} /$ (exemple, /gada/ ou /gaga/) ; pour les environnements vocaliques de faible aperture nous nous sommes limités à 12 non-mots $\mathrm{C}_{1} \mathrm{~V}_{1} \mathrm{C}_{2} \mathrm{~V}_{2}$ comportant deux consonnes identiques $\left(\mathrm{C}_{1}=\mathrm{C}_{2}=/ \mathrm{p}, \mathrm{t}, \mathrm{k}, \mathrm{b}, \mathrm{d}, \mathrm{g} /\right.$ et $\mathrm{V}_{1}=\mathrm{V}_{2}=/ \mathrm{i}, \mathrm{u} /$, par exemple, / $\mathrm{kiki} /$ ou $/ \mathrm{gugu} /$ ) pour des raisons de durée de tâche, qui aurait été trop longue et trop fatigante pour nos sujets les plus âgés si toutes les alternatives avaient été présentées. Les items ont été préalablement enregistrés par une locutrice francophone avec une intonation neutre. Ils ont été présentés en ordre aléatoire au sujet à l'aide d'un ordinateur PC portable à travers un casque, et il lui était demandé de les répéter. Les productions des participants ont été enregistrées à l'aide d'un enregistreur audio portable Zoom H5 avec couple stéréo en $\mathrm{X} / \mathrm{Y}$. Les analyses acoustiques ont été réalisées à l'aide du logiciel Praat. Les VOT a été défini pour chaque consonne comme la durée de l'intervalle temporel entre le début de l'explosion de la consonne et le premier cycle périodique de la voyelle. La présence de voisement était objectivée par l'observation d'une périodicité sur le signal. Nous avons également calculé le pourcentage de voisement de chaque consonne voisée (proportion de la durée de périodicité sur le signal lors du prévoisement de la consonne voisée par rapport à la durée complète de la consonne, commençant au début du prévoisement jusqu'au relâchement de l'occlusion).

\subsection{Procédure}

Les participants ont été évalués individuellement à leur domicile dans un local calme. Nous leur avons présenté les tâches sur 3 jours différents afin de ne pas les fatiguer. Chaque séance durait environ 30 minutes. L'ordre des tâches était le suivant : Jour 1 : (1) Anamnèse, (2) Dénomination d'images (40 premiers items), (3) Répétition de non-mots VOT (24 premiers items), (4) Désignation de phrases ; Jour 2 : (1) Dénomination d'images (fin), (2) Répétition de non-mots VOT (fin) ; Jour 3 : (1) Évaluation des fonctions exécutives, (2) Audiométrie tonale.

\section{Résultats}

La Figure 1 représente les valeurs moyennes des VOT pour les 4 groupes de participants, en fonction du lieu d'articulation et du voisement des occlusives, pour la première $\left(\mathrm{C}_{1}\right)$ et deuxième consonne $\left(\mathrm{C}_{2}\right)$. Nous avons effectué deux ANOVAS mixtes sur les valeurs des VOT des deux consonnes avec un design 4 (groupes : 40-49, 50-59, 60-69 et 70-79) x 2 (voisement : voisée ou non voisée) x 3 (lieu d'articulation : labial, dental, palatal).

Pour $\mathrm{C}_{1}$, les VOT moyens apparaissent, dans chaque groupe, bien différenciés en fonction du trait de voisement, comme le confirme l'effet principal de voisement, $F(3,2257)$ $=5140.74, p<.001$. Les valeurs de VOT correspondant à des réalisations de phonèmes voisés présentent des valeurs clairement négatives indiquant la réalisation d'un prévoisement ; celles qui correspondent à la réalisation d'un phonème non voisé présentent des valeurs positives. L'effet du lieu d'articulation (labial vs. dental vs. palatal) est également significatif, $F(3,2257)=9.73, p<.001$. Pour les consonnes non voisées, le VOT croît régulièrement au fur et à mesure que l'articulation se postériorise ; pour les consonnes voisées, dans les groupes de participants entre 50 et 79 ans, la palatale /g/ présente un VOT plus long que les deux autres consonnes /b/ et /d/. Dans le groupe des 40 à 49 ans, la labiale /b/ présente un VOT plus court que les deux autres occlusives voisées. Bien que les structures générales soient similaires dans les trois groupes de sujets, la variable de groupe 
d'âge exerce également un effet significatif, $F(3,2257)=9.73, p<.001$. De même que l'interaction âge x voisement, $F(3,2257)=9.73, p<.001$. L'effet de l'âge se manifeste principalement par une réduction graduelle, avec l'âge, de la différenciation (néanmoins toujours nette) entre les VOT des phonèmes voisés et ceux des phonèmes non voisés ; celleci s'opère principalement par diminution de la durée du prévoisement, le VOT des consonnes non voisées évoluant peu, de ce point de vue. Les autres interactions (âge x lieu d'articulation et âge $\mathrm{x}$ lieu $\mathrm{x}$ voisement) ne sont pas significatives.

Dans la $\mathrm{C}_{2}$, nous n'observons pas cet effet de l'âge, $F(3,2257)=1.85$, $n s$, mais bien des effets du voisement, $F(3,2257)=23.36, p<.001$ et du lieu d'articulation, $F(3,2257)=$ 5094.24, $p<.001$. Par contre, aucune interaction n'est significative, ce qui indique que l'âge ne semble pas avoir d'influence sur le voisement ou le lieu d'articulation dans la $\mathrm{C}_{2}$. Ainsi, la distinction entre les voisées et non voisées est présente dans chaque groupe et similaire entre les groupes d'âges. Pour les non voisées, nous observons des valeurs de VOT plus grandes pour la palatale $/ \mathrm{k} /$ que pour les occlusives $/ \mathrm{t} / \mathrm{et} / \mathrm{p} /$. Pour les voisées, nous notons des VOT plus longs pour les labiales /b/ que pour les occlusives /d/ et /g/.

C1.

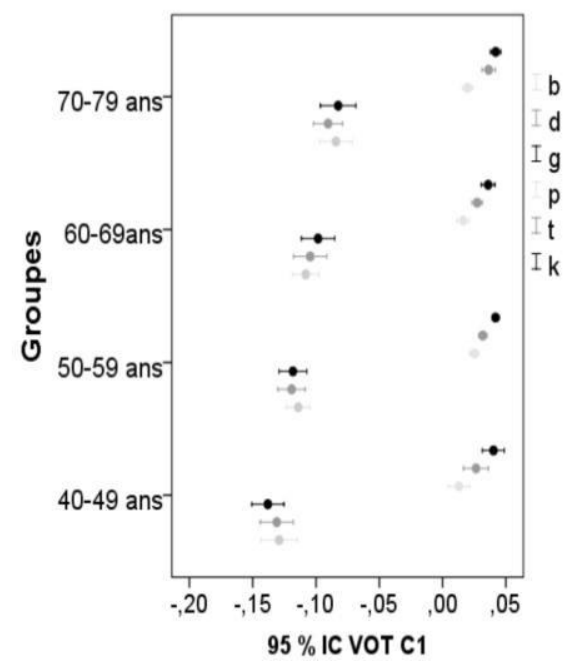

C2.

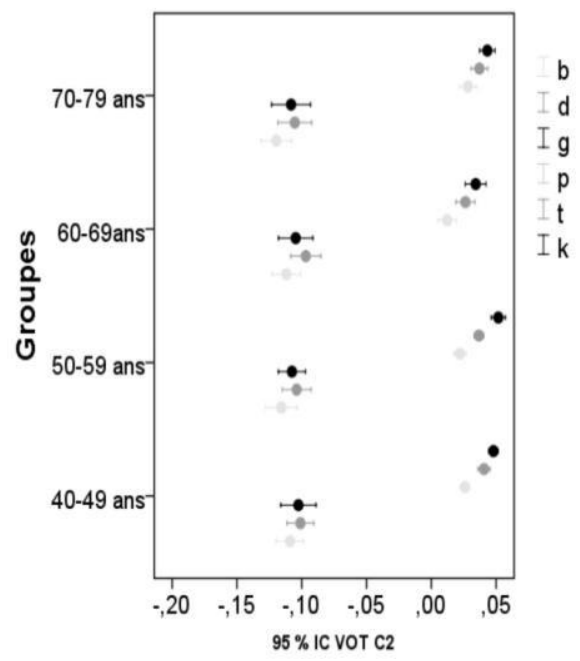

Fig. 1. Valeurs des VOT dans les 4 groupes de participants, en fonction de l'occlusive attendue. Pour la Consonne $1(\mathrm{C} 1)$ et la Consonne 2 (C2).

Nous nous sommes ensuite intéressés aux pourcentages de voisement des consonnes 1 et 2 voisées. Ceci est représenté dans la Figure 2. Les ANOVAS réalisées sur le pourcentage de voisement des $\mathrm{C}_{1}$ et $\mathrm{C}_{2}$ montre un effet de l'âge au niveau des deux consonnes $\left(\mathrm{C}_{1}: F(3,1124)=7.17, p<.001 ; \mathrm{C}_{2}: F(3,1126)=23.06, p<.001\right)$. Pour la $\mathrm{C}_{1}$, le test post-hoc de Tuckey $(p<.05)$ indique que le pourcentage de voisement diminue significativement chez les participants de plus de 60 ans. Pour la $\mathrm{C}_{2}$, le test post-hoc de Tuckey $(p<.05)$ montre que les participants de 70-79 et 40-49 présentent des pourcentages de voisement moins importants que dans les groupes de 50-59 et 60-69 qui ne diffèrent pas entre eux. 
C1

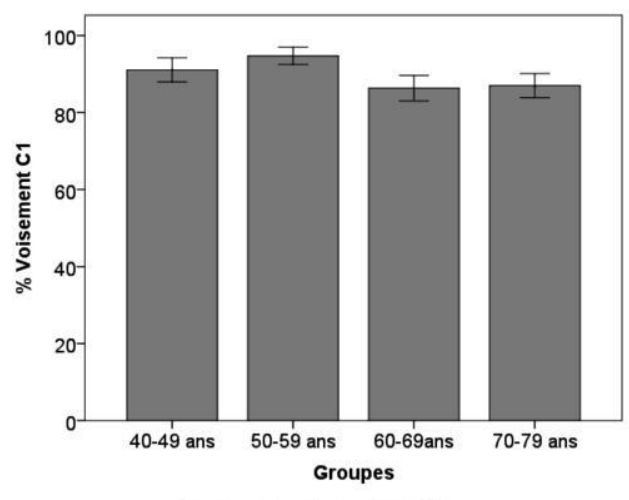

Barres d'erreur Intervalle de confiance à $95 \%$
$\mathrm{C} 2$

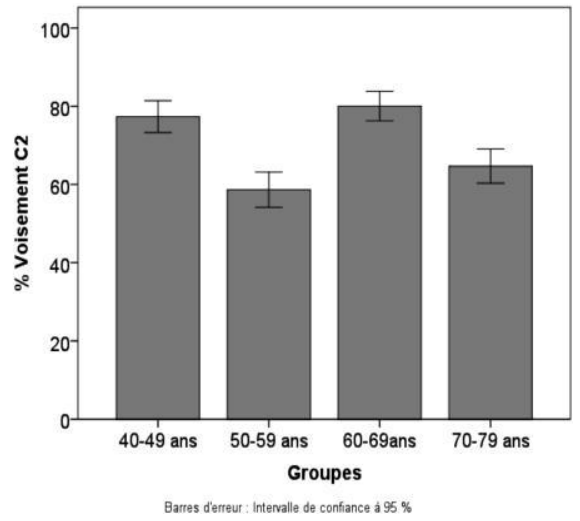

Fig. 2. Pourcentages de voisement des occlusives voisées 1 (C1) et 2 (C2) dans les 4 groupes de participants.

Enfin, étant donné que certaines études ont montré que la durée des VOT pouvait être influencée par le débit, et que celui-ci est ralenti chez les personnes âgées (Schötz, 2007b), nous avons également calculé la durée totale des non-mots, afin d'obtenir une estimation du débit des participants. Les durées moyennes des non-mots sont présentées dans le Tableau 2. L'ANOVA effectuée sur les durées des non-mots indique une augmentation des durées des non-mots avec l'âge, $F(3,2257)=22.04, p<.001$. Le test post-hoc de Tuckey $(p<.05)$ indique que les personnes âgées présentent une durée des non-mots plus longue que les participants plus jeunes, qui ne diffèrent pas entre eux.

Tableau 2. Durées moyennes de production des non-mots (ms)

\begin{tabular}{|l|l|l|}
\hline Groupes & Moyenne & Ecart-type \\
\hline $40-49$ ans & 473.23 & 124.73 \\
\hline $50-59$ ans & 467.50 & 118.62 \\
\hline $60-69$ ans. & 451.64 & 119.46 \\
\hline $70-79$ ans & 489.14 & 121.30 \\
\hline
\end{tabular}

\section{Discussion}

Le but de cette recherche était d'explorer les effets du vieillissement sur les capacités de production de la parole en français, les études en la matière centrées sur la population francophone demeurant, aujourd'hui encore, fort rares. Nous nous sommes focalisés sur le délai d'établissement du voisement (VOT) parce que sa production nécessite un contrôle précis du timing articulatoire entre les articulateurs laryngés et supra-laryngés et qu'il est dès lors particulièrement intéressant dans les études auprès de participants présentant des difficultés de contrôle de la parole, comme les personnes âgées (Aoki \& Fukuoa, 2010; Bilodeau-Mercure \& Tremblay, 2016; Linville, 2004; Schötz, 2007b; Torre \& Barlow, 
2009; Tremblay et al., 2017). De plus, nous nous sommes intéressés aux effets de l'âge sur le VOT dans différentes décennies, entre 40 et 80 ans, afin d'étudier la logique d'évolution d'un éventuel déclin.

Les résultats obtenus dans notre étude vont dans le sens de la présence de difficultés au niveau de la production du VOT, comme montré principalement dans des études réalisées auprès de participants anglophones (Gates et al., 2010; Linville, 2004; Morris, 1989; Schötz, 2007; Sweeting \& Baken, 1982; Torre \& Barlow, 2009; Xuanda et al., 2018), mais ici auprès de sujets francophones. Au niveau de la $\mathrm{C}_{1}$, les résultats indiquent que tous les participants effectuent une distinction entre les occlusives non voisées et voisées au niveau du VOT, caractérisé par des valeurs de VOT positives pour les non voisées et négatives pour les voisées. Pour les consonnes non voisées, les valeurs de VOT augmentent au fur et à mesure que le lieu d'articulation se postériorise, comme cela a déjà été démontré dans d'autres études (Auzou et al., 2000; Cho \& Ladefoged, 1999), et on ne note pas de différence en fonction de l'âge. Par contre, en ce qui concerne les occlusives voisées, on note un effet de l'âge allant dans le sens d'une tendance globale à la diminution des valeurs de VOT, qui devient statistiquement significative après 70 ans. Ceci engendre une diminution de la différence entre les voisées et non voisées (dont les VOT restent stables), même si celle-ci reste présente dans tous les groupes d'âge. Pour la $\mathrm{C}_{2}$, les résultats sont différents. En effet, pour les non voisés, à l'instar de la $C_{1}$, on observe des valeurs de VOT plus grandes au fur et à mesure que l'articulation devient postérieure. Par contre, on ne note pas de valeurs de VOT plus courtes pour les voisées chez les participants plus âgés, et la distinction entre les voisées et non voisées reste la même dans tous les groupes d'âge. Enfin, les résultats indiquent également des pourcentages de voisement plus courts pour les $\mathrm{C}_{1}$ voisées après 60 ans, mais à nouveau pas pour la $\mathrm{C}_{2}$.

L'ensemble de ces résultats semble indiquer des difficultés à la fois pour initier le voisement des occlusives voisées mais également pour le maintenir après l'avoir initié. En effet, les consonnes occlusives voisées du français comportent un prévoisement relativement long (70 à $100 \mathrm{~ms}$ ), qui demande une coordination précise entre les articulateurs à la fois pour initier et maintenir ce voisement. Le fait que cela affecte les premières occlusives du non-mot mais pas les secondes renforce cette hypothèse, étant donné que le voisement est déjà initié pour la seconde consonne par la voyelle précédente. Ces difficultés affecteraient les personnes dès 60 ans.

Par contre, les VOT plus courts ne semblent pas être reliés à une vitesse d'élocution plus rapide, comme cela a pu être démontré dans la littérature (Schötz, 2007). En effet, les mesures de durées des non-mots indiquent que les personnes de plus de 70 ans présentent des durées de non-mots plus longues, suggérant une vitesse d'élocution plus ralentie, alors que la durée des VOT diminue avec l'âge.

Ces résultats rejoignent ceux de Ryalls et al. (1997), qui avaient montré la présence de valeurs de VOT plus courtes chez certains participants francophones de plus de 60 ans. Les résultats de notre étude corroborent cette observation. De plus, les difficultés pour maintenir le voisement peuvent aussi être reliées à d'autres études, réalisées en langue anglaise sur des voyelles, qui ont montré que les participants présentaient une plus grande instabilité pour maintenir le voisement après 60 ans, par exemple lors d'épreuves de tenue de voyelles (Bilodeau-Mercure, Kirouac, et al., 2015; Bilodeau-Mercure \& Tremblay, 2016; Ramig, 1983, 1986; Torre \& Barlow, 2009). Par contre, ceci n'a jamais été démontré pour des consonnes voisées française, à notre connaissance. Ces difficultés ont été reliées à des changements physiologiques de la structure oro-laryngée, comme une ossification des cartilages, une atrophie des muscles, à une instabilité du mécanisme laryngé, ou encore à une dégradation du contrôle moteur de la parole. Ces hypothèses pourraient expliquer les résultats observés dans notre étude. Cependant, des études futures devraient les confirmer, en utilisant d'autres techniques de mesures en plus des mesures acoustiques. 
Enfin, il est à noter qu'une difficulté liée au vieillissement pourrait également avoir interféré avec nos résultats. Il s'agit de la presbyacousie présentée par nos participants de plus de 70 ans. En effet, d'autres études ont indiqué des effets délétères de troubles auditifs sur la production du VOT, par exemple chez les participants présentant une surdité soudaine, même si la perte auditive observée chez les sujets dans ces études était plus importante que celle présentée par nos sujets (Perkell, Lane, Svirsky, \& Webster, 1992). Selon Auzou et al. (2000); Torre et Barlow (2009); Tremblay (2013), le déclin auditif lié à l'âge entrainerait une réduction du feed-back sensoriel qui contrôle la production de la parole, engendrant une diminution de la précision et de la stabilité de la parole. Ceci pourrait affecter le VOT et dès lors les résultats dans notre étude. Nous projetons d'y revenir dans des études futures. En outre, certaines études n'ont pas indiqué d'effets de la perte auditive sur le VOT. En effet, Ryalls et Larouche (1992) n'ont pas montré de différence significative sur les durées des VOT observées chez des enfants francophones présentant une surdité en comparaison avec des enfants entendants appariés en âge.

En conclusion, notre étude indique la présence d'un effet de l'âge sur la production de la parole, dans une population francophone, langue dans laquelle ce type d'étude est très rare. Le déclin observé dans notre étude affecte les durées des VOT et les pourcentages de voisement des consonnes occlusives voisées du français, principalement pour les premières consonnes des non-mots CVCV, suggérant des difficultés d'initiation et de maintien du voisement des occlusives voisées. Ces difficultés semblent apparaître très graduellement, même avant la soixantaine, âge où elles deviennent indubitables.

Comme nous l'avons souligné d'entrée, l'augmentation de l'âge moyen de la population mondiale rend de plus en plus nécessaire la connaissance précise des effets de l'âge sur les capacités communicatives des individus, et donc, notamment sur l'évolution de leurs aptitudes phoniques sous conditions de vieillissement, dans un contexte de qualité de vie satisfaisante. La mise au jour de données précises de ce type sur des phénomènes d'ordinaire considérés comme déjà bien connus (on ne considère généralement pas les occlusives du français comme terra incognita, et pourtant...) est probablement l'une des urgences de notre temps, à tout le moins pour ce qui concerne l'espace francophone.

Nous tenons à remercier Amélie Visentini et Jérémy Pouliart pour leur aide dans la collecte des données.

Nous souhaitons également remercier le Dr Quinette et le Dr Majerus pour la mise à disposition d'une partie du matériel d'évaluation.

\section{Références}

Aoki, T., \& Fukuoa, Y. (2010). Finger Tapping Ability in Healthy Elderly and Young Adults. Medicine \& Science in Sports \& Exercise, 42(3), 449-455. https://doi.org/10.1249/MSS.0b013e3181b7f3e1

Auzou, P., Ozsancak, C., Morris, R. J., Mary Jan, F. E., Hannequin, D., Jan, M., ... Hannequin, D. (2000). Voice onset time in aphasia , apraxia of speech and dysarthria : a review. Clinical Linguistics \& Phonetics, 14(2), 131-150. https://doi.org/10.1080/026992000298878

Baltes, P. B., Staudinger, U. M., \& Lindenberger, U. (1999). Lifespan psychology: Theory and Application to Intellectual Functioning. Annual Review of Psychology, 50(1), 471-507. https://doi.org/10.1146/annurev.psych.50.1.471

Baqué, L., Marczyk, A., Rosas, A., \& Estrada, M. (2015). Disability, repair strategies and communicative effectiveness at the phonic level: evidence from a multiple-case study. Neuropsycholinguistic Perspectives on Language Cognition, (May), 144-165. https://doi.org/10.4324/9780203797365 
Belleville, S., Rouleau, N., \& Caza, N. (1998). Effect of normal aging on the manipulation of information in working memory. Memory \& Cognition, 26(3), 572-583.

Bilocq, V., De Partz, M.-P., De Wilde, V., Pillon, A., \& Seron, X. (2001). Lexis. Test pour le diagnostic des troubles lexicaux chez le patient aphasique (De Boeck). Brussels (Belgium).

Bilodeau-Mercure, M., Kirouac, V., Langlois, N., Ouellet, C., Gasse, I., \& Tremblay, P. (2015). Movement sequencing in normal aging: speech, oro-facial, and finger movements. Age (Dordrecht, Netherlands), 37(4), 9813. https://doi.org/10.1007/s11357-015-9813-x

Bilodeau-Mercure, M., Lortie, C. L., Sato, M., Guitton, M. J., \& Tremblay, P. (2015). The neurobiology of speech perception decline in aging. Brain Structure \& Function, 220(2), 979-997. https://doi.org/10.1007/s00429-013-0695-3

Bilodeau-Mercure, M., \& Tremblay, P. (2016). Age Differences in Sequential Speech Production: Articulatory and Physiological Factors. Journal of the American Geriatrics Society, 64(11), e177-e182. https://doi.org/10.1111/jgs.14491

Burke, D. M., MacKay, D. G., Worthley, J. S., \& Wade, E. (1991). On the tip of the tongue: What causes word finding failures in young and older adults? Journal of Memory and Language, 30(5), 542-579. https://doi.org/10.1016/0749-596X(91)90026-G

Burke, D. M., \& Shafto, M. A. (2004). Aging and Language Production. Current Directions in Psychological Science, 13(1), 21-24. https://doi.org/10.1111/j.09637214.2004.01301006.x

Cho, T., \& Ladefoged, P. (1999). Variation and universals in VOT: evidence from 18 languages. Journal of Phonetics, 27, 207-229.

https://doi.org/10.1006/jpho.1999.0094

Connor, L. T., Spiro, A., Obler, L. K., \& Albert, M. L. (2004). Change in Object Naming Ability During Adulthood. The Journals of Gerontology Series B: Psychological Sciences and Social Sciences, 59(5), P203-P209. https://doi.org/10.1093/geronb/59.5.P203

Cruickshanks, K. J., Wiley, T. L., Tweed, T. S., Klein, B. E., Klein, R., Mares-Perlman, J. A., \& Nondahl, D. M. (1998). Prevalence of hearing loss in older adults in Beaver Dam, Wisconsin. The Epidemiology of Hearing Loss Study. American Journal of Epidemiology, 148(9), 879-886. https://doi.org/10.1093/oxfordjournals.aje.a009713

Gates, G. A., Gibbons, L. E., McCurry, S. M., McCusrry, S. M., Crane, P. K., Feeney, M. P., \& Larson, E. B. (2010). Executive dysfunction and presbycusis in older persons with and without memory loss and dementia. Cognitive and Behavioral Neurology: Official Journal of the Society for Behavioral and Cognitive Neurology, 23(4), 218223. https://doi.org/10.1097/WNN.0b013e3181d748d7

Joanette, Y., Nespoulous, J.-L., \& Roch Lecours, A. (1998). MT 86 - Protocole MontréalToulouse d'examen linguistique de l'aphasie (Ortho Edit). Isebergues.

Krampe, R. T., Mayr, U., \& Kliegl, R. (2005). Timing, Sequencing, and Executive Control in Repetitive Movement Production. Journal of Experimental Psychology: Human Perception and Performance, 31(3), 379-397. https://doi.org/10.1037/00961523.31.3.379

Linville, S. E. (2000). The aging voice. In R. D. Kent \& M. J. Ball (Eds.), Voice quality measurement (Singular T, pp. 359-376). San Diego, CA.

Linville, S. E. (2004). The aging voice. The American Speech-Language-Hearing Association (ASHA), 12-21. Leader.

Lisker, L., \& Abramson, a S. (1964). A cross-language study of voicing in initial stops: Acoustical measurements. Word Journal Of The International Linguistic Association, Vol. 20, pp. 384-422. https://doi.org/10.1080/00437956.1964.11659830

Marczyk, A., Baqué, L., Rosas, A., \& Nespoulous, J. L. (2011). On the nature of speech errors in aphasia: Acoustic analysis of the speech output of 8 native speakers of 
spanish with aphasia. Procedia - Social and Behavioral Sciences, 23(September 2015), 84-85. https://doi.org/10.1016/j.sbspro.2011.09.181

Maylor, E. A., Vousden, J. I., \& Brown, G. D. (1999). Adult age differences in short-term memory for serial order: data and a model. Psychology and Aging, 14(4), 572-594.

Morris, R. J. (1989). Vot and dysarthria: A descriptive study. Journal of Communication Disorders, 22(1), 23-33. https://doi.org/10.1016/0021-9924(89)90004-X

Morris, R. J., McCrea, C. R., \& Herring, K. D. (2008). Voice onset time differences between adult males and females: Isolated syllables. Journal of Phonetics, 36(2), 308-317. https://doi.org/10.1016/j.wocn.2007.06.003

Nespoulous, J. L., Baqué, L., Rosas, A., Marczyk, A., \& Estrada, M. (2013). Aphasia, phonological and phonetic voicing within the consonantal system: preservation of phonological oppositions and compensatory strategies. Language Sciences, 39(1), 117-125. https://doi.org/https://doi.org/10.1016/j.langsci.2013.02.015

Oberauer, K. (2001). Removing irrelevant information from working memory: a cognitive aging study with the modified Sternberg task. Journal of Experimental Psychology. Learning, Memory, and Cognition, 27(4), 948-957. Retrieved from http://www.ncbi.nlm.nih.gov/pubmed/11486928

Perkell, J., Lane, H., Svirsky, M., \& Webster, J. (1992). Speech of cochlear implant patients: a longitudinal study of vowel production. The Journal of the Acoustical Society of America, 91(5), 2961-2978.

Quinette, P., Laisney, M., Lambert, J., Bocoyran, S., Eustache, F., \& Desgranges, B. (2014). Working memory (WM) and executive functions (EF) in aphasic patients. Annals of Physical and Rehabilitation Medicine, 57(S1), 139. https://doi.org/10.1016/j.rehab.2014.03.1573

Ramig, L. A. (1983). Effects of physiological aging on vowel spectral noise. Journal of Gerontology, 38(2), 223-225. Retrieved from http://www.ncbi.nlm.nih.gov/pubmed/6827040

Ramig, L. A. (1986). Aging speech: Physiological and sociological aspects. Language \& Communication, 6(1-2), 25-34. https://doi.org/10.1016/0271-5309(86)90003-0

Ryalls, J., Cliche, A., Fortier-blanc, J., Coulombe, I., \& Prud'hommeaux, A. (1997). Voiceonset time in younger and older French-speaking Canadians. Clinical Linguistics \& Phonetics, 11(3), 205-212. https://doi.org/10.3109/02699209708985191

Ryalls, J., Provost, H., \& Arsenault, N. (1995). Voice onset time production in Frenchspeaking aphasics. Journal of Communication Disorders, 28(1), 205-215.

Salthouse, T. A. (2009). When does age-related cognitive decline begin? Neurobiology of Aging, 30(4), 507-514. https://doi.org/10.1016/j.neurobiolaging.2008.09.023

Schmidt, R., Freidl, W., Fasekas, F., Reinhart, B., \& Grieshofer, P. (1994). Mattis Dementia Rating Scale.

Schötz, S. (2007). Acoustic analysis of adult speaker age. Speaker Classification I. Lecture Notes in Computer Science, Vol 4343, 88-107. https://doi.org/10.1007/978-3-54074200-5 5

Serniclaes, W. (1987). Etude expérimentale de la perception du trait de voisement des occlusives du français. Free university of Brussels.

Surprenant, A. M. (2007). Effects of Noise on Identification and Serial Recall of Nonsense Syllables in Older and Younger Adults. Aging, Neuropsychology, and Cognition, 14(2), 126-143. https://doi.org/10.1080/13825580701217710

Sweeting, P M, \& Baken, R. J. (1982). Voice onset time in normal-aged population. Journal of Speech and Hearing Research, 25(1), 129-134.

Sweeting, Patricia M., \& Baken, R. J. (1982). Voice Onset Time in a Normal-Aged Population. Journal of Speech Language and Hearing Research, 25(1), 129. https://doi.org/10.1044/jshr.2501.129

Torre, P., \& Barlow, J. A. (2009). Age-related changes in acoustic characteristics of adult 
speech. Journal of Communication Disorders, 42(5), 324-333.

https://doi.org/10.1016/j.jcomdis.2009.03.001

Tremblay, P., Sato, M., \& Deschamps, I. (2017). Age differences in the motor control of speech: An fMRI study of healthy aging. Human Brain Mapping, 38(5), 2751-2771. https://doi.org/10.1002/hbm.23558

Verhaegen, C., Delvaux, V., Fagniart, S., Huet, K., Piccaluga, M., \& Harmegnies, B.

(2019). Phonological and phonetic impairment in aphasic speech: an acoustic study of the voice onset time of six French-speaking aphasic patients. Clinical Linguistics \& Phonetics, 1-21. https://doi.org/10.1080/02699206.2019.1619095

Verhaegen, C., \& Poncelet, M. (2013). Changes in Naming and Semantic Abilities With Aging From 50 to 90 years. Journal of the International Neuropsychological Society, 19(02), 119-126. https://doi.org/10.1017/S1355617712001178

Xuanda, C., Ziyu, X., \& Jian, H. (2018). The Trajectory of Voice Onset Time with Vocal Aging. Interspeech Conference, 1556-1560.

https://doi.org/10.21437/Interspeech.2018-60 